\title{
A REMARK ON IRREDUCIBLE REPRESENTATIONS OF LIE ALGEBRAS
}

\author{
JU. A. BAHTURIN \\ (Received 27 June 1978; revised 8 November 1978) \\ Communicated by R. Lidl
}

\begin{abstract}
In addition to the results of the paper (Bachturin (1974)) we give the precise form of the necessary and sufficient conditions ensuring that all irreducible representations of a Lie algebra were of finite bounded degree.
\end{abstract}

Subject classification (Amer. Math. Soc. (MOS) 1970): 17 B 35.

\section{Preliminaries}

In my paper (Bachturin (1974)) necessary and sufficient conditions are given on a Lie algebra $\mathrm{g}$ over a field $k$ of prime characteristic $p$ in order that the universal envelope $U(\mathfrak{g})$ of $g$ satisfy a nontrivial identical relation. The corollary of the main theorem concerning degrees of irreducible representations of Lie algebras is, however, rather obscure. The aim of the present note is to give its precise form. In fact, the following theorem holds:

THEOREM. Let $k$ be an algebraically closed field of characteristic $p>0$ and let $\mathfrak{g}$ be a Lie algebra over $k$. All irreducible representations of $\mathfrak{g}$ are of finite bounded dimension if, and only if,

(i) $\mathfrak{g}$ possesses an abelian ideal $\mathfrak{h}$ of finite codimension;

(ii) all inner derivations ad $x, x \in g$, are algebraic;

(iii) $\operatorname{dim}_{k}(\mathfrak{h})<\operatorname{Card}(k)$.

COROLLARY. If, in addition, $k$ is countable, then $\mathfrak{g}$ is finite dimensional. 
REMARK. The case of a base field $k$ of characteristic zero is essentially simpler. In this case necessary and sufficient conditions follow from a theorem of Latyšev (1963) and Lemma 1 below.

Proposition. Let $\mathrm{g}$ be a Lie algebra over an algebraically closed field of characteristic 0 . All irreducible representations of $\mathfrak{g}$ are of finite bounded dimension if, and only if, $\mathrm{g}$ is abelian and $\operatorname{dim}_{k}(\mathrm{~g})<\operatorname{Card}(k)$.

For all the basic facts concerning Lie algebras the reader is referred to Jacobson (1962) or to the more recent book of Dixmier (1974).

We denote by Card $(S)$ the cardinality of a set $S$. Given a Lie algebra $g$ and an element $x \in \mathrm{g}$ we denote by $\operatorname{ad} x$ the $\operatorname{map}(\operatorname{ad} x)(y)=[x, y], y \in \mathrm{g}$.

\section{Two lemmas}

We start with the simpler

LEMMA 1. Let $k$ be an algebraically closed field, $X$ some set of indeterminates and $A=k[X]$ a polynomial ring over $k$. Then $A$ possesses a nonzero infinite dimensional irreducible representation if, and only if, $\operatorname{Card}(X) \geqslant \operatorname{Card}(k)$.

Proof. Note first that if $M$ is a simple module over $A$ then $M \cong A / I$ where $I$ is some maximal ideal of $A$, that is, $M$ is a commutative field extending $k$.

Thus, if $X$ is finite, then $M$ is a field finitely generated over $k$ as a ring. In this case by Hilbert's Nullstellensatz (Cohn (1977), p. 429) $M$ is algebraic over $k$ and hence $M=k$. Therefore $X$ may be assumed infinite and, obviously,

$$
\operatorname{dim}(M)=\operatorname{dim}(A / I) \leqslant \operatorname{dim}(A)=\operatorname{Card}(X) .
$$

Consider next an element $u \in A / I$ and let $B=k(u)$ be the subfield of $A / I$ generated by $u$. If $u$ is transcendental over $k$, then $\operatorname{dim}(B)=\operatorname{Card}(k)$. Indeed $k(u)$ contains a subsystem $\left\{(u-\alpha)^{-1}, \alpha \in k\right\}$ which is linearly independent and has cardinality equal to that of $k$, so that $\operatorname{dim}(B) \geqslant \operatorname{Card}(k)$. The converse inequality holds since $B$ as a ring is generated by the system $R=\left\{(u-\alpha)^{-1},(u-\beta)(u-\gamma)^{-1}, \alpha, \beta, \gamma \in k\right\}$ whose cardinality also coincides with $\operatorname{Card}(k)$.

Thus, if $A$ possesses an infinite dimensional irreducible representation in $M=A / I$ but $\operatorname{Card}(X)<\operatorname{Card}(k)$ then any element $u \in A / I$ is algebraic over $k$, that is, $\operatorname{dim}_{k}(A / I)=1$, which is a contradiction. 
If, on the contrary, $\operatorname{Card}(X) \geqslant \operatorname{Card}(k)$, then any mapping of $X$ onto $R$ extends to an epimorphism of $A=k[X]$ onto $B=k(u)$, thus proving that $A$ possesses an infinite dimensional irreducible representation.

LEMMA 2. Assume that the base field $k$ has prime characteristic $p$ and is algebraically closed. If $\mathrm{g}$ is a Lie algebra over $k$ satisfying the conditions (i) and (ii) of the main theorem then $\mathrm{g}$ possesses an infinite dimensional irreducible representation if, and only if, such a representation exists for $\mathfrak{h}$.

Proof. Let $e_{1}, e_{2}, \ldots, e_{n}$ form a basis of $g$ modulo $\mathfrak{h}$ over $k$. By Lemma 1.5 from Bachturin (1974) there exist polynomials $f_{1}, f_{2}, \ldots, f_{n}$ such that the elements $z_{1}=f_{1}\left(e_{1}\right), z_{2}=f_{2}\left(e_{2}\right), \ldots z n=f_{n}\left(e_{n}\right)$ are central in $U=U(\mathrm{~g})$. Consider then a subalgebra $R$ of $U(\mathfrak{g})$ generated by $z_{1}, z_{2}, \ldots z_{n}$ together with $U(\mathfrak{h})$. Since $z_{1}, z_{2}, \ldots, z_{n}$ are central, $R$ is abelian. Moreover, since $U(\mathrm{~g})$ is a free left $U(\mathfrak{h})$-module with the monomials $e_{1}^{m_{1}} \ldots e_{n}^{m_{n}}\left(\sum m_{i} \geqslant 0\right)$ as basis, it follows that $R$ is isomorphic to a polynomial ring $k\left[z_{1}, z_{2}, \ldots, z_{n}, u_{\alpha}, \alpha \in B\right]$ where $\left\{u_{\alpha}, \alpha \in B\right\}$ is a basis of $\mathfrak{h}$ over $k$. Since, obviously, only the case of infinite $B$ need be considered, $R$ is certainly isomorphic with $U(\mathfrak{h})$, the polynomial algebra $k\left[u_{\alpha}, \alpha \in B\right]$. This shows that $R$ possesses an infinite dimensional irreducible representation if, and only if, $U(\mathfrak{h})$ (or $\mathfrak{h}$ ) does.

Now assume that $\mathfrak{h}$ has no infinite dimensional irreducible representation. By Lemma $1, \operatorname{dim}_{k}(\mathfrak{h})<\operatorname{Card}(k)$. In this case $U(\mathfrak{g})$ is a module of finite type over $R$ whose dimension is equal to that of $\mathfrak{h}$, the dimension of $U(\mathfrak{g})$ over $k$ is the same as $\operatorname{dim}(\mathfrak{h})$, and by the preceding $\operatorname{dim}(U(\mathfrak{g}))<\operatorname{Card}(k)$.

Any simple $U(\mathrm{~g})$-module is isomorphic to some $U(\mathrm{~g}) / I$, where $I$ is a maximal left ideal of $U(\mathrm{~g})$. Let $B$ be the largest subalgebra of $U(\mathrm{~g})$ containing $I$ and such that $I$ is a two-sided ideal in $B$. Then $K=B / I$ is a skew field and by a corollary of the density theorem (Cohn (1977), p. 337) either $\operatorname{dim}_{K}(U(\mathrm{~g}) / I)$ is finite or for each $n$ the matrix ring $K_{n}$ is a homomorphic image of some subalgebra of $U(\mathrm{~g})$. But by the main theorem of Bachturin (1974) $U(\mathrm{~g})$ satisfies a nontrivial identity which therefore holds in $K_{n}$ for each $n$, and this is of course impossible (see Cohn (1977), p. 455).

Thus it remains to consider the case $\operatorname{dim}_{K}(U(\mathrm{~g}) / I)<\infty$. But then in fact $K=k$, for $K$ is algebraic over $k$. Indeed, choose $u \neq 0$ in $K$. Then assuming $u$ transcendental over $k$, one immediately sees (as in the previous lemma) that $\operatorname{dim}(k(u))=\operatorname{Card}(k)$ which contradicts our former assumption (in fact,

$$
\operatorname{dim}(k(u)) \leqslant \operatorname{dim}(K) \leqslant \operatorname{dim}(B / I) \leqslant \operatorname{dim}(U(\mathrm{~g}) / I) \leqslant \operatorname{dim}(U(\mathrm{~g}))<\operatorname{Card}(k)) .
$$

The converse part of the lemma is even simpler. Let $V$ be an infinite dimensional simple left $R$-module. Then considering $U(\mathrm{~g})$ as a right $R$-module one may form a 
left $U(\mathfrak{g})$-module $W$ by putting

$$
W=U(\mathrm{~g}) \otimes_{R} V .
$$

Clearly one may identify $V$ with its canonical image $1 \otimes V$ in $W$. Take some nonzero element $v \in V$ and consider the cyclic $U(\mathrm{~g})$-submodule $V^{\prime}$ generated by $v$. Let $M$ be a maximal $U(\mathrm{~g})$-submodule of $W$ which does not contain $v$ (its existence trivially follows by Zorn's lemma). From the $R$-irreducibility of $V$ it follows that $M \cap V=\{0\}$. Consider next the $U(\mathrm{~g})$-submodule $V^{\prime \prime}=V^{\prime}+M$. Evidently, $T=V^{\prime \prime} / M$ is an irreducible $U(\mathrm{~g})$-module. On the other hand, bearing in mind the $R$-module structure of $W$ one has

$$
V \cong V / V \cap M \cong V+M / M \subset V^{\prime}+M / M=V^{\prime \prime} / M=T,
$$

which proves that $T$ is infinite dimensional over $k$.

The proof of the lemma is now complete.

\section{Proofs of the theorem and the proposition}

Assume first that $\mathrm{g}$ is a Lie algebra all of whose irreducible representations have finite bounded degrees. It is well known (use, for example, Cohn (1977), p. 348) that the universal envelope $U(\mathrm{~g})$ is semiprimitive, that is, the intersection of all primitive ideals of $U(\mathrm{~g})$ equals zero. Therefore $U(\mathrm{~g})$ may be represented as a subdirect product of its primitive homomorphic images having by our assumption bounded dimensions. Thus $U(\mathrm{~g})$ satisfies a nontrivial polynomial identity and one may apply either the main theorem of Bachturin (1977) stating that in this case $g$ satisfies the conditions (i) and (ii) from the introduction, or the theorem of Latysev (1963) stating that $g$ is abelian, provided the base field $k$ has characteristic 0 .

The remaining condition follows from Lemma 2 when the base field has characteristic $p>0$ or Lemma 1 otherwise.

The same lemmas as well trivially prove the converse implications of the theorem and the proposition. For, by these lemmas $g$ cannot possess an infinite dimensional irreducible representation. If, further, $M$ is a finite dimensional simple $g$-module such that $\operatorname{dim}_{k}(M)=n$, then by the density theorem and since $k$ is algebraically closed, one immediately deduces that $k_{n}$ is an epimorphic image of $U(\mathrm{~g})$. Since $U(g)$ satisfies a nontrivial identity, this trivially bounds $n$ and proves the whole theorem. 


\section{References}

Ju. A. Bachturin (1974), 'Polynomial identities in the universal envelopes of Lie algebras', $J$. Austral. Math. Soc. 18, 10-21.

P. M. Cohn (1977), Algebra (John Wiley and Sons, London).

N. Jacobson (1962), Lie algebras (John Wiley and Sons, New York).

J. Dixmier (1974), Algèbres enveloppantes (Gauthier-Villars, Paris).

V. N. Latyšev (1963), 'Two remarks on PI-algebras', Sibirsk. Mat. Ž. 4, 1120-1121.

Department of Mechanics and Mathematics

Moscow State University

117234 Moscow

USSR

Current address:

Department of Mathematics

Bedford College

Regent's Park, London NW1 4NS

England 